\title{
What we don't know about eccentric viewing
}

T W Raasch

\section{The link between central scotomas, Troxler fading, and preferred retinal locus}

E very clinician who has worked with patients with central scotomas has observed the difficulty those patients experience. Not only have they lost the ability to resolve fine detail, they also see nothing "straight ahead". The resulting behaviour, most commonly called "eccentric viewing", typically involves the development of a "pseudofovea", or a preferred retinal locus (PRL). The use of a PRL is primarily an eye movement control issue: the individual must be able to place an object of interest on a particular fundus location. In addition to eye position control, however, successful use of an eccentric location must involve much more than just that. As Deruaz and colleagues have pointed out in this issue of $B J O(\mathrm{p} 461)$, higher level sensory processes are likely to be involved.

These authors have made the observation that some patients alternate between two retinal locations when deciphering a word. On further questioning, these patients reported that the displayed word was more distinct immediately after an eye movement. That phenomenon is attributed to the Troxler effect-that is, the fading of an object under stabilised conditions. This observation prompted the authors to conduct a laboratory experiment with normally sighted observers. This experiment is easy enough to replicate as a demonstration. Such a demonstration does seem to support the suggestion that Troxler fading might be experienced by those with central scotomas. We do not know, however, whether Troxler fading contributes to the development of multiple PRLs. As Deruaz et al suggest, saccades and other eye movements that occur naturally, even with a single PRL, probably prevent Troxler fading.

The efficient use of a PRL requires careful eye movement control. It must also require a shift of one's attention to that location in the visual field. Consider a patient with two PRLs reading a full page of text. Words will fall on both PRLs simultaneously much of the time. In order to make sense of what is being viewed, that patient must be able to focus attention selectively on one or the other PRL. Without that ability, both regions of text would be equally salient.

An area that remains relatively unexplored is binocularity and PRLs. Most of what is known about PRL use applies to monocular viewing conditions. In an individual with bilateral central scotomas, how is the input from the two eyes integrated? Do PRLs tend to develop at corresponding retinal locations? Does a PRL tend to develop at a location that corresponds to a scotomatous area in the other eye? If PRLs develop in both eyes at non-corresponding locations,

\section{Myopia}

\section{Myopia in Asia}

\section{P J Foster}

\section{An unexploded bomb}

a n this issue of the BJO (p 538), Saw and colleagues show that, after exclusion of syndrome associated and retinopathy of prematurity (ROP) related myopia, there was no identifiable link between birth weight and refractive error in later childhood. Low birth weight has been linked to adult risk of cardiovascular disease (CVD), hypertension, diabetes, and cancer. what happens under binocular conditions? Can both PRLs at non-corresponding points be used in a reading task? Can that person shift attention between those PRLs? Can this person experience confusion as the result of input from both PRLs? Is the input from one eye simply suppressed? We do not know the answers to those questions.

Eccentric viewing training is part of many comprehensive visual rehabilitation programmes. One wonders what should be the goal of eccentric viewing training. Does one reinforce to the trainee that the eccentric point is eccentric, preserving the normal oculocentric direction? Or does one attempt to replace the non-functioning fovea with that eccentric point? That is, should the goal be to shift the oculocentric direction to that eccentric point? If that is possible, it seems that would produce the most effective and effortless eccentric viewing behaviour possible. If the oculocentric direction shifts, however, is it a binocular shift, or does the shift occur independently (and perhaps unequally) for both eyes?

How one learns to view objects in the presence of a central scotoma is a complex issue. We have learned a great deal about this problem, but there is much more that we do not know. Perhaps we will learn whether training helps patients develop skills they otherwise would not develop, what type of training might be most effective, and in which patients training will be most effective. The growing number of visually impaired individuals will be better served when we have better answers to these questions.

Br J Ophthalmol 2004;88:443.

doi: 10.1136/bjo.2003.034827

\section{Author's affiliation}

T W Raasch, College of Optometry, 320 W

10th Avenue, Columbus, OH, USA;

traasch@optometry.osu.edu

The effects of low birth weight are increased by slow infant growth and rapid weight gain in later childhood. ${ }^{1}$ The so called "Barker hypothesis" suggests that antenatal factors may "program" physiology in later life. The absence of a clear association with refractive error suggests the process coordinating ocular dimensions does not fall under the control of a similar mechanism. Put simply, they show that bigger children have bigger eyes, but not necessarily (after correction for other socioeconomic factors) higher levels of myopia. However, it has been shown that children who are born small have small eyes, and that this 
trait is retained in adulthood. Fledelius examined 70 subjects with low birth weight (less than $2000 \mathrm{~g}$ ) and 67 full term controls. The low birth weightocular size deficit remained an adult feature, even in seemingly normal eyes. There was also a parallel, permanent lack of "catch up" in height, head circumference, and other anthropometric factors. ${ }^{2}$

What marks this paper as interesting is the analytical method used by the investigators. Previous epidemiological studies of refractive error have used refraction as the sole end point. ${ }^{34}$ However, there is a growing recognition that the risk factors for, and natural history of, myopia and hypermetropia cannot be fully understood without recourse to ocular biometry. ${ }^{5-7}$ The inclusion of biometric data in the analysis has strengthened the authors' abilities to examine the determinants of refractive error in this study of 1413 schoolchildren in Singapore. With the recognition that that bigger people have bigger eyes, without necessarily higher rates of myopia, the importance of correcting for co-determinants of axial dimensions, such as height, can be readily appreciated. ${ }^{8}$ Similarly, in the study of adult refractive error, changes in lens opacity are often accompanied by changes in refractive index, further complicating the modelling of risk factors. ${ }^{?}$

The size of the problem may parallel the rapid industrialisation and economic growth in China
The aetiology of myopia still excites considerable debate. Comparison of prevalence data between Asia and the West suggests substantially higher rates of myopia in industrialised regions of east Asia. It is tempting to propose a genetic basis for this observation. However, Saw and colleagues have also shown that greater reading exposure is associated with myopia in Singapore. ${ }^{10}$ This, once again, raises the "chicken and egg" debate of cause and effect, although there is a broad consensus that near work is causally linked to myopia. ${ }^{11}$ None the less, it is becoming more plausible that myopia has a genetic predisposition with environmental triggers, the interaction of which results in phenotypic plasticity. Studies of myopia genetics have concentrated almost exclusively on high myopia, with inconclusive results to date. Lower levels of myopia are likely to be an even more challenging proposition. "Common" myopia probably has a complex genetic mechanism for which Mendelian models of disease will be inadequate. Myopia will probably become one of the most fruitful areas of ophthalmology and visual science for the study of the interaction of genetic and environmental mechanisms in causation of disease.

The prevalence of myopia in east Asia appears to be increasing at an alarming rate. It seems likely that with the size of the problem will parallel the rapid industrialisation and economic growth in China. If this issue is not tackled immediately, we risk ignoring a ticking bomb.
Br J Ophthalmol 2004;88:443-444.

doi: 10.1136/bjo.2003.031476

\section{Author's affiliation}

P J Foster, Division of Epidemiology, Institute of Ophthalmology, University College London, London ECIV 9EL, UK; p.foster@ucl.ac.uk

\section{REFERENCES}

1 Barker DJP, Eriksson JG, Forsen T, et al. Fetal origins of adult disease: strength of effects and biological basis. Int J Epidemiol 2002;31:1235-9.

2 Fledelius HC. Ophthalmic changes from age of 10 to 18 years. A longitudinal study of sequels to low birth weight. IV. Ultrasound oculometry of vitreous and axial length. Acta Ophthalmol 1982;60:403-11.

3 Katz J, Tielsch JM, Sommer A. Prevalence and risk factors for refractive errors in an adult inner city population. Invest Ophthalmol Vis $\mathrm{SCi}$ 1997:38:334-40.

4 Attebo K, lvers RQ, Mitchell P. Refractive errors in an older population: the Blue Mountains Eye Study. Ophthalmology 1999;106:1066-72.

5 Lin LL, Shih YF, Hsiao CK, et al. Epidemiologic study of the prevalence and severity of myopia among schoolchildren in Taiwan in 2000. J Formosa Med Assoc 2001;100:684-91.

6 Zadnik K, Manny RE, Yu JA et al. Ocular component data in schoolchildren as a function of age and gender. Optom Vis Sci 2003;80:226-36

7 Lo PI, Ho PC, Lau JT, et al. Relationship between myopia and optical components - a study among Chinese Hong Kong student population. Yan Ke Xue Bao 1996; 12:121-5.

8 Wong TY, Foster PJ, Johnson GJ, et al. The relationship between ocular dimensions and refraction with adult stature: the Tanjong Pagar Survey. Invest Ophthalmol Vis Sci 2001;42:1237-42.

9 Wong TY, Foster PJ, Ng TP, et al. Variations in ocular biometry in an adult Chinese population in Singapore: The Tanjong Pagar Survey. Invest Ophthalmol Vis Sci 2001;42:73-80.

10 Saw SM, Chua WH, Hong CY, et al. Nearwork in early-onset myopia. Invest Ophthalmol Vis Sci 2002;43:332-9.

11 Goss DA. Nearwork and myopia. Lancet 2000;356:1456-7.

\section{The pathogenesis of diabetic retinopathy}

\section{R Stanford}

\section{Sticky blood or sticky vessels, or both}

$\mathrm{T}$ here has been considerable interest in the past few years in the early pathological events that lead to vascular occlusion in diabetic retinopathy. The finding of increased leucostasis (leucocytes attached to the endothelial wall) is a common pathological event in both human disease ${ }^{12}$ and in experimental models. ${ }^{3}{ }^{4}$ The heightened leucocyte/endothelial interaction induced by hyperglycaemia occurs very early in the diabetic process and, as a result, endothelial dysfunction and subsequent apoptosis occur. Although there is some reserve in terms of endothelial division and proliferation, this becomes exhausted with time, leading to the appearance of acellular capillary tubes, the pathological hallmark of disease. ${ }^{5}$ These tubes do not support blood flow and retinal ischaemia supervenes. Presumably there is pre-capillary arteriolar thrombosis due to the loss of the anti-thrombogenic endothelial lining, but pathological evidence for this is scant. ${ }^{6}$ The question arises as to whether this process is mediated by changes in leucocytes (sticky blood), changes in the endothelial surface (sticky vessels), or both.

Supporting the "sticky blood" hypothesis are the findings that leucocytes (particularly polymorphonuclear cells) are less deformable in diabetes, ${ }^{7}$ that there is upregulation of integrins (ligands for vascular adhesion molecules) on their surface, ${ }^{8}$ and that they adhere more strongly to cultured endothelial cells in vitro in both static assays and under conditions of flow. ${ }^{9}$ Furthermore, in vivo treatment with anti-integrin monoclonal antibodies experimentally markedly reduces leucostasis. ${ }^{4}$ Recent work has shown that the glycosylating enzyme, core 2 GlcNAc transferase, activity is upregulated in leucocytes derived from diabetic 
patients and that this upregulation positively correlates with the presence of established retinopathy. This enzyme causes post-translational modifications of the O-glycans on the cell membrane, some of which are molecules involved with endothelial interaction, and specific blockade of the phosphorylation of this enzyme abrogated the heightened leucocyte-endothelial interaction in vitro. ${ }^{1011}$

Support for abnormalities in the endothelium (sticky vessel) comes from the immunohistochemical demonstration of increased adhesion molecule expression (particularly intercellular adhesion molecule-1, ICAM-1) on the cell surface in response to hyperglycaemia in both human ${ }^{1}$ and experimental models, ${ }^{3}$ and that the observed leucostasis in experimental models could be partially prevented by treatment with a monoclonal antibody against ICAM-1. Moreover, ICAM-1 knockout mice made diabetic do not develop the expected retinal vascular changes evident in their wild type counterparts. ${ }^{5}$ The cause of this heightened expression is not known but may be from the action of endogenous vascular endothelial growth factor (VEGF) acting through a nitric oxide pathway since treatment with aptamers to VEGF reduced retinal leucostasis and blood-retinal barrier breakdown in experimental models. ${ }^{12} 13$

\section{Abnormalities of both blood and vessel wall probably contribute to the process of vaso-obliteration in diabetes}

What occurs as a result of this leucocyte-endothelial interaction is not certain. Under normal physiological conditions, the interaction is necessary to allow the immune system to sample the microvascular environment with the leucocyte rapidly returning to the circulation if the correct sequential expression of adhesion molecules to allow firm tethering to the endothelial surface is not found. In diabetes, the reduced deformability and increased stickiness of the leucocyte may be sufficient to physically block capillary tubes. ${ }^{14}$ More likely is that the prolonged interaction leads to endothelial dysfunction and apoptosis, eventually leading to complete loss of the capillary lining..$^{15} 16$

One of the principal hypotheses on which the above scenario is basednamely, that of increased adhesion molecule expression in the diabetic retinal vasculature, is challenged in this issue of the BJO. In a carefully performed immunohistochemical study of human specimens, Hughes et al (p 566) show, in contrast with previous studies, that there is little difference in the expression of ICAM-1 between normal and diabetic retinas. Furthermore, their studies also show diffuse ICAM-1 staining of neural retina; this was increased in the diabetic specimens and correlated with local breakdown of the bloodretinal barrier. The reason for the disparity in their results and those of previous studies is comprehensively discussed and, although the diabetic history of their specimens is not known, the evidence is sufficiently forceful to prompt a re-evaluation of the subject.

It seems likely that abnormalities of both blood and vessel wall contribute to the process of vaso-obliteration in diabetes although the relative importance of either still remains to be determined. Further work examining the role of reactive oxygen species in the promotion of a prothrombotic phenotype in the microvasculature and the contribution of leucocyte-platelet interactions to microthrombus formation needs to be done. Understanding of the pathophysiological basis of disease is fundamental to the formulation of new treatments. Already, considerable interest has been shown in the use of antiinflammatory drugs in the amelioration of diabetic retinopathy ${ }^{17}$ and early reports of the use of intravitreal steroids are promising. Certainly, there is now an increasing rationale for the use of specific protein kinase C inhibitors. ${ }^{11} 1819$ Current advances in knowledge of the pathology of the disease are likely to throw up further candidates in the near future.

Br J Ophthalmol 2004;88:444-445. doi: $10.1136 /$ bjo.2003.031443

\section{Author's affiliation}

M R Stanford, Medical Eye Unit, St Thomas's Hospital, Lambeth Palace Road, London SE 1 7EH, UK; miles.stanford@kcl.ac.uk

\section{REFERENCES}

1 McLeod DS, Lefer DJ, Merges C, et al. Enhanced expression of intercellular adhesion molecule-1 and $\mathrm{p}$-selectin in the diabetic human retina and choroid. Am J Pathol 1995; 147:642-53.

2 Lutty GA, Cao J, McLeod DS. Relationship of polymorphonuclear leukocytes to capillary dropout in the human diabetic choroid. Am J Pathol 1997;151:707-14.

3 Miyamoto K, Khosrof S, Bursell S-E, et al. Prevention of leukostasis and vascular leakage in streptozotocin-induced diabetic retinopathy via intercellular adhesion molecule-1 inhibition. Proc Natl Acad Sci 1999:96:10836-41.

4 Canas-Barouch FC, Miyamoto K, Allport JR, et al. Integrin-mediated neutrophil adhesion and retinal leukostasis in diabetes. Invest Ophthalmol Vis Sci 2000;41:1153-8.

5 Adamis AP. Is diabetic retinopathy an inflammatory disease? $\mathrm{Br} J$ Ophthalmol 2002;86:363-5.

6 Garner A. Histopathology of diabetic retinopathy in man. Eye 1993:7:250-3.

7 Miyamoto K, Ogura Y, Kenmochi S, et al. Role of leukocytes in diabetic microcirculatory disturbances. Microvasc Res 1997;54:43-8.

8 Rao KMK, Hatchell DL, Cohen HJ, et al. Alterations in stimulus-induced integrin expression in peripheral blood neutrophils of patients with diabetic retinopathy. Am J Med Sci 1997;313:131-7.

9 Morigi M, Angioletti S, Imberti B, et al. Leukocyteendothelial interaction is augmented by high glucose concentrations and hyperglycaemia in a NF-kB-dependent fashion. J Clin Invest 1998;101:1905-15

10 Chibber R, Ben-Mahmud BM, Coppini D, et al. Activity of the glycosylating enzyme, core 2 GlcNAc $(\beta 1,6)$ transferase, is higher in polymorphonuclear leukocytes from diabetic patients compared with age-matched control subjects. Diabetes 2000;49:1724-30.

11 Chibber R, Ben-Mahmud BM, Mann GE, et al. Protein kinase $c \beta 2$-dependent phosphorylation of core 2 GlcNAc-T promotes leukocyte-endothelial cell adhesion. Diabetes 2003;52:1519-27.

12 Joussen AM, Poulaki V, Qin W, et al. Retinal vascular endothelial growth factor induces intercellular adhesion molecule 1 and endothelial nitric oxide synthase expression and initiates early diabetic retinal leukocyte adhesion in vivo. Am J Pathol 2002;160:501-9.

13 Ishida S, Usui T, Yamashiro K, et al. VEGF 164 is proinflammatory in the diabetic retina. Invest Ophthalmol Vis Sci 2003:44:2155-62.

14 Schröder S, Palinki W, Schmid-Schönbein GW. Activated monocytes and granulocytes, capillary nonperfusion, and neovascularization in diabetic retinopathy. Am J Pathol 1991;139:81-100.

15 Mizutani M, Kern TS, Lorenzi M. Accelerated death of retinal microvascular cells in human and experimental diabetic retinopathy. J Clin Invest 1996;97:2883-90.

16 Joussen AM, Murata T, Tsujikawa A, et al. Leukocyte-mediated endothelial cell injury and death in the diabetic retina. Am J Pathol 2001; 158: 147-52.

17 Joussen AM, Poulaki V, Mitsiades N, et al. Nonsteroidal anti-inflammatory drugs prevent early diabetic retinopathy via TNF- $\alpha$ suppression. Faseb J 2002;16:438-40.

18 Nonaka A, Kiryu J, Tsujikawa A, et al. PKC- $\beta$ Inhibitor (LY333531) attenuates leukocyte entrapment in retinal microcirculation of diabetic rats. Invest Ophthalmol Vis Sci 2000;41:2702-6.

19 Abiko T, Abiko A, Clermont AC, et al. Characterization of retinal leukostasis and hemodynamics in insulin resistance and diabetes. Diabetes 2003;52:829-37. 